Mots. Les langages du politique

$111 \mid 2016$

Normes et usages de la langue en politique

\title{
Femen. La reconnaissance médiatique d'un féminisme aux seins nus
}

Femen, media recognition of naked breasts feminism

Femen, el reconocimiento mediático de un topless feminismo

\section{Marion Dalibert et Nelly Quemener}

\section{OpenEdition \\ Journals}

Édition électronique

URL : https://journals.openedition.org/mots/22373

DOI : $10.4000 /$ mots. 22373

ISBN : 978-2-84788-836-2

ISSN : 1960-6001

Éditeur

ENS Éditions

\section{Édition imprimée}

Date de publication : 10 septembre 2016

Pagination : 83-102

ISBN : 978-2-84788-835-5

ISSN : 0243-6450

\section{Référence électronique}

Marion Dalibert et Nelly Quemener, «Femen. La reconnaissance médiatique d'un féminisme aux seins nus », Mots. Les langages du politique [En ligne], 111 | 2016, mis en ligne le 10 septembre 2018,

consulté le 23 avril 2022. URL : http://journals.openedition.org/mots/22373 ; DOI : https://doi.org/ $10.4000 /$ mots. 22373

\section{(C) ENS Éditions}




\section{Femen. La reconnaissance médiatique d'un féminisme aux seins nus}

Collectif féministe ukrainien né en 2008, Femen bénéficie d'une couverture de presse plus ou moins régulière en France durant ses premières années d'existence en Ukraine et qui s'intensifie lorsque le collectif s'implante dans l'hexagone au cours de l'été 2012. Une telle couverture interroge à plusieurs niveaux, tant elle dissone avec la médiatisation souvent discrète des mouvements sociaux. Tout d'abord, elle invite à porter attention aux processus de médiatisation et d'événementialisation, étant considéré que ces derniers sont le produit d'une coconstruction entre le mouvement et les journalistes. Si la médiatisation relève d'un choix opéré dans les rédactions sur ce qui va faire l'objet d'un article (Molotch, Lester, 1996), elle ne peut être dissociée des actions même du mouvement, voire de ses stratégies d'accès aux médias et à l'espace public. On peut ainsi voir dans les actions collectives de Femen ce que Patrick Champagne appelle des «manifestations de papier» (1990), soit des actions en partie destinées à être photographiées et filmées par les journalistes. Proches du happening et de la performance, elles sont marquées d'un sceau particulièrement identifiable : les activistes, auréolées d'une couronne de fleurs, agissent poitrine nue et parée de slogans, faisant de leurs corps de véritables «corpsdiscours» (Paveau, 2013). Les tentatives de Femen pour attirer l'attention des médias se révèlent néanmoins singulières en ce qu'elles sont indissociables et même constitutives de ses actions et revendications. Le collectif porte en effet une cause très générale, la lutte contre le patriarcat, mais se saisit de «fenêtres d'opportunités politiques et discursives» (Cefaï, 2007, p.490), à l'instar des débats sur l'ouverture du mariage pour les personnes de même sexe, pour donner corps à ses revendications et préciser les contours de l'oppression, tout en assurant son maintien à l'agenda médiatique (Lefébure, Lagneau, 2001, p. 80). Au-delà de la relation du mouvement aux médias, la forte couverture médiatique de Femen invite à rendre compte des pendants de cette visibilité et des logiques de reconnaissance qu'elle implique dans la «sphère publique globale » (Fraser,

Université Charles-de-Gaulle-Lille 3, GERiiCO

marion.dalibert@gmail.com

Université Sorbonne Nouvelle-Paris 3, CIM, équipe MCPN

nellyquemener@gmail.com

Mots. Les langages du politique $\mathrm{n}^{\circ} 111$ juillet $2016 \bullet 83$ 
2001)1. Ainsi, alors que Femen n'est pas l'unique collectif féministe à utiliser ce répertoire d'actions², il est le seul à bénéficier d'une telle couverture de presse. En étudiant la présence du collectif dans la presse française, il s'agit par conséquent d'interroger tout à la fois la couverture médiatique de Femen comme le résultat d'une véritable «lutte pour la visibilité » du mouvement lui-même (Voirol, 2005), et comme le fruit d'un encodage du mouvement par les journalistes selon des critères de légitimité sociale. Autrement dit, que nous dit la médiatisation de Femen des formes acceptées et valorisées du féminisme?

Pour répondre à cette question, nous proposons l'analyse d'un corpus comprenant l'ensemble des articles parus sur Femen d'octobre 2009 (date du premier article publié en France) à décembre 2013 dans la presse française nationale généraliste (Le Parisien-Aujourd'hui en France, La Croix, L'Express, Le Figaro, L'Humanité, Libération, Le Monde, Le Nouvel Observateur, Le Point) et spécialisée (Les Échos, Les Inrockuptibles, Paris Match, La Tribune), ce qui fait, en totalité, 270 articles. En empruntant des méthodes au champ de l'analyse de discours, nous avons porté notre attention sur le processus d'événementialisation : il s'est agi de comptabiliser le nombre d'articles parus par mois ainsi que leur taille (en nombre de mots), afin d'observer les périodes où les activistes ont été les plus visibles dans les médias. Nous avons ensuite collecté et trié les syntagmes servant à désigner et définir les membres de Femen, les victimes de sexisme et les coupables mis en scène dans le récit médiatique, et observé leur évolution dans le temps. Cette étude quantitative des «paradigmes désignationnels et définitionnels » (Mortureux, 1993) a permis de rendre compte de régularités dans la construction médiatique du mouvement en fonction des différentes phases de médiatisation. Elle s'accompagne d'un versant qualitatif qui s'attache à mettre au jour et contextualiser les querelles d'interprétation et les conflits idéologiques qui accompagnent les différentes actions. L'enjeu est ici de saisir la manière dont les médias donnent sens au mouvement, au travers des lexiques et répertoires qui construisent autant de «chaînes de signification » et déterminent les régimes du visible et du dicible (Hall, 2007).

L'analyse critique de la couverture médiatique de Femen permet de mettre au jour trois temps significatifs de la « reconnaissance sociale » 3 dont a bénéficié le collectif dans la sphère publique : la période ukrainienne, d'octobre 2009 à juillet 2012, significative d'une altérisation et hypersexualisation du collec-

1. Nancy Fraser définit cette sphère publique matérialisée par les grands médias comme un espace de conflictualité et de lutte dans lequel les groupes subordonnés œuvrent pour faire entendre leurvoix et participer à la définition des problèmes publics.

2. «La Barbe », par exemple, utilise également le happening : les activistes s'insèrent dans les lieux de pouvoir en arborant une barbe pour dénoncer, par l'ironie, l'hégémonie masculine.

3. La reconnaissance sociale est un acte d'attribution de valeur et de qualités effectué par un individu/groupe sur un autre (Honneth, 2000). Celle-ci est toujours une expression publique qui se manifeste dans les médias d'information par un traitement journalistique à la fois important et positif (Voirol, 2005). 
Graphique 1 : Nombre d'articles parus par mois (tous journaux confondus) lorsque Femen est territorialisé en Ukraine (octobre 2009 - juillet 2012)

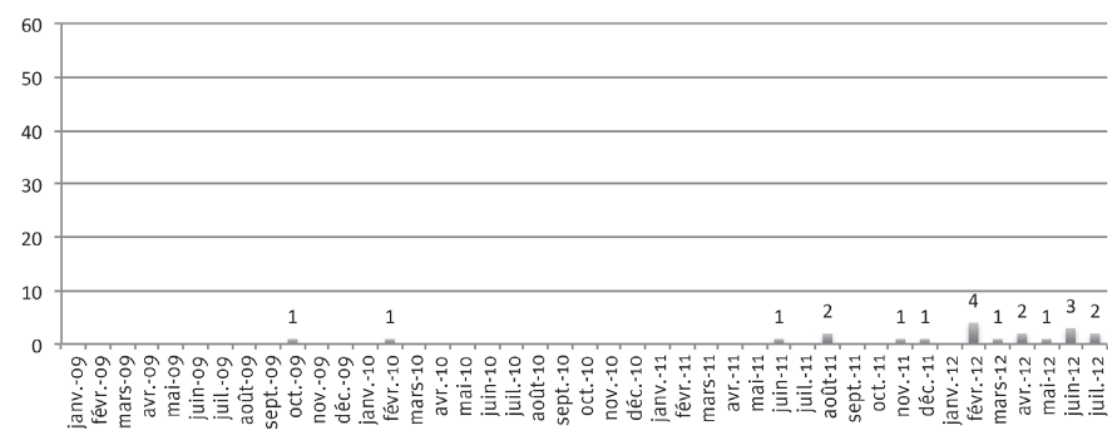

tif ; la territorialisation du mouvement en France, d'août 2012 à mars 2013, marquée par l'élévation de Femen au rang d'acteur du débat public et une extension des domaines de la lutte féministe; le transfert du combat en Tunisie avec l' " affaire Amina », d'avril 2013 à août 2013, caractérisé par l'ethnoracialisation des victimes et des responsables du sexisme ainsi que par la création d'un féminisme « universaliste». Chacune de ces phases de la médiatisation se structure autour de chaînes d'oppositions duales qui s'imbriquent et assoient l'« avant-gardisme » du mouvement face à l'« archaïsme » de ses cibles : émancipation/assujettissement, démocratie/corruption, femmes blanches dénudées/musulmanes voilées, laïcité/religion, Europe de l'Ouest/Europe de l'Est, blanchité immaculée/blanchité salie, Occident/Pays musulmans. Comme nous le verrons, ces oppositions dessinent symboliquement de nouvelles frontières territoriales sur la base de différences ethnoraciales, et participent de la valorisation d'une certaine conception de l'émancipation associée à la «blanchité» [whiteness] (Dyer, 1997; Cervulle, 2013). Elles s'accompagnent en outre d'une transformation dans l'image et l'identité médiatique du mouvement. Perçues au départ comme ukrainiennes, les Femen font l'objet d'une ethnoracialisation sensible dans la sexualisation des corps féminins. Une fois le mouvement territorialisé en France, elles se trouvent associées par les journalistes à une parole d'autorité qui participe à l'ethnoracialisation des responsables désignés, souvent lointains, du sexisme. À travers l'analyse de tels déplacements, nous défendrons que la couverture médiatique de Femen associe le sexisme à des territoires «autres».

\section{La période ukrainienne, entre exotisme et altérisation}

La médiatisation de Femen est précoce. Sur les 270 articles du corpus, vingt sont publiés d'octobre 2009 à juillet 2012, soit avant l'arrivée de Femen en 
France, évoquant la vie - et surtout les difficultés - des activistes en Ukraine (graphique 1). Même si cette première publicité provient majoritairement de la presse sociohistoriquement identifiée à gauche (Libération, Le Monde, L'Humanité, Les Inrockuptibles, Le Nouvel Observateur), à l'électorat traditionnellement sensible à la cause des minorités (Bonnafous, 1991, p. 35), elle étonne car, rares sont les mouvements sociaux à faire l'objet d'articles dans la presse nationale (Fillieule, Tartakowsky, 2008, p.134), d'autant plus lorsque les actions s'ancrent à l'étranger et sont éloignées des rédactions (Neveu, 1999, p. 39). Or, si Femen semble, dès cette période, doté de ressources internes et d'outils de communication tel un site web permettant d'attirer les médias à l'échelle de l'Ukraine, rien ne laisse pour autant présager qu'il puisse faire l'objet d'une médiatisation en France. Aussi, quoique encore balbutiante et disparate, cette couverture journalistique est significative d'une certaine valorisation médiatique et témoigne d'un intérêt certain des journalistes français pour le mouvement.

Cette première phase se caractérise par la construction, dans les discours définitoires du collectif et de l'Ukraine, d'une rupture quasi «civilisationnelle» entre l'Europe de l'Ouest et l'Europe de l'Est. S'appuyant sur les propos des Femen, la presse associe l'Ukraine au «tourisme sexuel» (Libération, 2 octobre 2009), à « la prostitution galopante », au « recul de la démocratie» (L'Express, 10 août 2011) et à «la corruption, l'immobilisme gouvernemental » (Les Inrockuptibles, 7 mars 2012).

Sur la totalité des termes utilisés à cette période pour désigner les responsables de l'assujettissement des femmes, vingt-trois font référence à la prostitution et quinze aux gouvernements russe et ukrainien alors que la religion, qui constitue la cible principale du mouvement lorsque celui-ci s'implante en France, n'est mentionnée qu'à onze reprises (tableau 1). L'Ukraine est ainsi donnée à voir comme un pays autoritaire où, à l'image de l'ancien régime soviétique, les opposants politiques seraient surveillés, voire emprisonnés. Les membres de Femen sont présentés comme les premières victimes de cette absence de liberté, à travers des contrôles et des intimidations quotidiennes par la police, comme l'a mis en avant un envoyé spécial des Inrockuptibles le 10 septembre 2012 : «Le troisième jour de notre rencontre, trois agents des services secrets ukrainiens sont postés devant le local des Femen et surveillent les allées et venues de leurs visiteurs. » Le récit, fortement narré dans la presse, de la fuite d’Inna Shevchenko en France pour échapper à la police ukrainienne après avoir scié une croix à Kiev en soutien au Pussy Riot, nourrit la représentation de ce climat d'oppression.

La médiatisation du mouvement se structure autour d'une opposition entre une Ukraine archaïque et une Europe de l'Ouest qui, par contraste, apparaît comme résolument moderne. Cette opposition se nourrit d'une altérisation par les discours portés sur les habitants du pays, au travers notamment de la mise en scène de masculinités et de féminités d'un autre temps et de rapports de 
Tableau 1. Liste des principaux syntagmes désignant et définissant les responsables du sexisme dans la médiatisation de Femen

\begin{tabular}{|c|c|c|c|c|}
\hline & $\begin{array}{l}\text { Période ukrai- } \\
\text { nienne octobre } \\
2009 \text {-juillet } \\
2012\end{array}$ & $\begin{array}{l}\text { Implantation en } \\
\text { France } \\
\text { août } 2012 \text { - mars } \\
2013\end{array}$ & $\begin{array}{l}\text { "Affaire Amina " } \\
\text { avril - août } 2013\end{array}$ & $\begin{array}{l}\text { Baisse de la } \\
\text { médiatisation } \\
\text { septembre - } \\
\text { décembre } 2013\end{array}$ \\
\hline $\begin{array}{l}\text { Prostitution } \\
\text { /Tourisme } \\
\text { sexuel }\end{array}$ & 23 & 10 & 8 & 0 \\
\hline Gouvernants & $\begin{array}{l}\text { 1. (dont } \\
\text { 11 syntagmes } \\
\text { renvoient à } \\
\text { l'Ukraine et } 4 \text { à } \\
\text { la Russie) }\end{array}$ & $\begin{array}{l}\text { 1. (dont } \\
11 \text { syntagmes } \\
\text { renvoient à } \\
\text { l'Ukraine, } 2 \text { à la } \\
\text { Russie et } 2 \text { aux } \\
\text { pays musul- } \\
\text { mans) }\end{array}$ & $\begin{array}{l}\text { 4. (dont } \\
23 \text { renvoient à } \\
\text { la Tunisie, } 15 \text { à } \\
\text { la Russie et } 7 \text { à } \\
\text { l'Ukraine) }\end{array}$ & $\begin{array}{l}4 \text { (dont } \\
3 \text { renvoient à } \\
\text { l'Ukraine et } 1 \text { à } \\
\text { la Russie) }\end{array}$ \\
\hline Religion & $\begin{array}{l}\text { 1. (10 syn- } \\
\text { tagmes ren- } \\
\text { voient à la reli- } \\
\text { gion chrétienne } \\
\text { - catholique, } \\
\text { orthodoxe ou } \\
\text { protestante - et } \\
1 \text { à la religion } \\
\text { musulmane) }\end{array}$ & $\begin{array}{l}\text { 9. }(75 \text { syn- } \\
\text { tagmes font } \\
\text { référence à } \\
\text { la religion } \\
\text { chrétienne } \\
\text { - catholique, } \\
\text { orthodoxe ou } \\
\text { protestante -, } \\
8 \text { à la religion } \\
\text { musulmane et } \\
7 \text { à toutes les } \\
\text { religions) }\end{array}$ & $\begin{array}{l}\text { 17. (152 syn- } \\
\text { tagmes } \\
\text { renvoient à } \\
\text { la religion } \\
\text { musulmane, } \\
15 \text { à la religion } \\
\text { chrétienne } \\
\text { - orthodoxe ou } \\
\text { protestante - et } \\
5 \text { à toutes les } \\
\text { religions) }\end{array}$ & $\begin{array}{l}\text { 2. (dont } \\
21 \text { syntagmes } \\
\text { renvoient à } \\
\text { la religion } \\
\text { chrétienne } \\
\text { - catholique, } \\
\text { orthodoxe ou } \\
\text { protestante - et } \\
1 \text { à la religion } \\
\text { musulmane) }\end{array}$ \\
\hline $\begin{array}{l}\text { "Les } \\
\text { hommes» }\end{array}$ & $\begin{array}{l}1 \text { (syntagme qui } \\
\text { fait référence } \\
\text { aux hommes } \\
\text { d'Europe de } \\
\text { l'Ouest) }\end{array}$ & $\begin{array}{l}\text { 1. (10 syn- } \\
\text { tagmes font } \\
\text { référence } \\
\text { aux hommes } \\
\text { d'Europe de } \\
\text { l'Ouest, } 6 \text { aux } \\
\text { Ukrainiens et } \\
2 \text { aux musul- } \\
\text { mans) }\end{array}$ & $\begin{array}{l}\text { 1. (dont } \\
13 \text { syntagmes } \\
\text { renvoient aux } \\
\text { musulmans et } \\
5 \text { aux Ukrai- } \\
\text { niens) }\end{array}$ & 0 \\
\hline $\begin{array}{l}\text { Extrême } \\
\text { droite }\end{array}$ & 0 & 5 & 30 & 13 \\
\hline Pays & $\begin{array}{l}1 \text { (syntagme qui } \\
\text { fait référence } \\
\text { aux pays musul- } \\
\text { mans) }\end{array}$ & $\begin{array}{l}4 \text { (syntagmes } \\
\text { qui font réfé- } \\
\text { rence aux pays } \\
\text { musulmans) }\end{array}$ & $\begin{array}{l}\text { 2. (syntagmes } \\
\text { qui renvoient } \\
\text { à la société tuni- } \\
\text { sienne) }\end{array}$ & 0 \\
\hline
\end{tabular}


genre inégalitaires, contraires aux valeurs supposées de l'Europe de l'Ouest. Défaillants, irresponsables et alcooliques, les Ukrainiens sont décrits comme incapables de satisfaire à leur rôle d'époux et de chef de famille : «Le mouvement [Femen] surgit en 2006 dans cette Ukraine rigide où les femmes portent à bout de bras la famille quand leurs hommes plongent dans la bouteille», écrit entre autres Le Nouvel Observateur (4 avril 2013). L'ensemble du schéma familial ukrainien se voit ici disqualifié : renvoyé à un modèle patriarcal, il contraindrait les femmes, une fois mariées, à des tâches traditionnellement attachées au féminin (s'occuper de leur mari et de leurs enfants), ainsi qu'au rôle de garant du foyer. À travers ces descriptions, la presse produit l'image d'hommes ukrainiens condamnables car responsables de l'assujettissement des femmes. Plus fortement encore, elle les renvoie au statut d' " autre » différent du «nous » (Berting, 2001), attendu qu'elle oppose une masculinité ukrainienne sexiste et défaillante à une figure idéalisée de la masculinité d'Europe de l'Ouest, égalitaire et responsable. Ce procédé de disqualification de la masculinité participe à un «marquage» (Brekhus, 2005) sociodiscursif de l'ethnicité.

Ce processus d'ethnoracialisation4 se traduit en outre par une «hypersexualisation »5 de l'image des jeunes femmes d'Europe de l'Est. C'est en effet par la représentation médiatique d'une (hyper)féminité associée à l'érotisme et à la séduction que les jeunes Ukrainiennes se voient caractérisées comme telles et différenciées des femmes françaises (Dalibert, 2014). Ce processus se structure néanmoins de façon ambivalente ${ }^{6}$. II renvoie, d'une part, à une série de connotations négatives, en ce qu'il sert à dépeindre l'univers de la prostitution auquel seraient condamnées les jeunes Ukrainiennes lorsqu'elles ne sont pas mariées : «Un seul pas peut [les] conduire à se prostituer. Il suffit de papoter avec un type qui distribue des flyers incitant des jeunes filles à travailler comme "hôtesses" " (Le Monde, 7 mars 2013). Mais cette hypersexualisation se matérialise d'autre part dans les descriptions particulièrement laudatives des membres de Femen, alors renvoyées à une série d'attributs physiques féminins et à leurdite beauté. Sacha Chevtchenko est ainsi qualifiée de «blonde comme le blé ukrainien, grande et photogénique », pouvant «facilement devenir mannequin et enchaîner les shootings de mode » (L'Express, 10 août 2011). Ces descriptions physiques s'accompagnent parfois d'une érotisation explicite, à l'instar de cet article de Libération (2 octobre 2009) où les activistes sont décrites

4. Ce processus rend compte de la construction de frontières symboliques entre les groupes par l'attribution de traits culturels différents, voire opposés (De Rudder, Poiret, Vourc'h, 2000, p. 31). Celle-ci, souvent associée à des caractéristiques physiques, conduit à naturaliser et à hiérarchiser les groupes et donc à symboliser l’idée - socialement construite - de race.

5. La sexualisation du corps féminin reste par ailleurs un phénomène identifié dans les médias (Gill, 2003).

6. Par structure ambivalente, Stuart Hall entend une représentation organisée autour de deux extrêmes - i.e. le Noir représenté sous les traits du «barbare » et du «noble sauvage», d'une part, du «traître » et du «fidèle serviteur», d'autre part (Hall, 1997b). 
comme "s'amusant de leurs charmes", ou encore celui des Inrockuptibles paru le 10 septembre 2012 évoquant la «moue boudeuse » d'Oksana Chatchko «allongée nonchalamment dans un coin du deux pièces qui fait office de QG ». Ces portraits, parce qu'ils mobilisent l'imaginaire d'une sexualité mystérieuse et mystifiée, rendent compte de la production d'un univers fantasmé, presque exotique, indissociable de l'ethnoracialisation du mouvement. Cette exotisation laudative des activistes participe à la production d'une figure idéalisée de la féminité, reposant sur la beauté, la blondeur, la blanchité, qui deviennent ici synonymes de "pureté » et de virginité (Dyer, 2004, p.122), et de son revers, celle d'une figure de prostituée, à la beauté, la blondeur et la blanchité «salies» par une sexualité débordante et active.

De telles projections se matérialisent visuellement dans les photographies publiées de Femen, contribuant alors à dépolitiser leur usage du corps. Lorsqu'elles posent, celles-ci crient et/ou adoptent un regard ferme et frontal, tout en reproduisant le salut au poing levé emprunté au Black Power. Ce geste militant, plutôt associé à la masculinité, rend compte de leur volonté de situer leur combat dans la filiation de mouvements sociaux historiques, tout en se construisant une image de féministes fortes et combatives. Or, même s'il se trouve au cœur de l'iconographie promue par Femen, il n'est jamais mentionné par les journalistes. Il est laissé à l'attention des lecteurs au sein de photographies qui, plutôt que d'insister sur le geste quasi viril, valorisent une féminité normative à travers la monstration d'une nudité circonscrite à certains corps. Les activistes montrées à l'image sont ainsi principalement celles qui répondent aux normes corporelles de la féminité blanche : elles sont jeunes, minces, blanches, épilées, généralement maquillées et ont les cheveux longs. Par la suite et, notamment, une fois le collectif formé en France, cette mise en scène photographique perdure. Alors que Femen compte une diversité de corporalités notamment visible sur sa page Facebook et souhaitée par les membres actifs du mouvement (Bouton, 2014), les photographies font disparaître les corps charnus, âgés ou androgynes ou les relèguent au deuxième, voire au troisième plan. On peut sans doute voir dans ces mises en scène photographiques le résultat d'une véritable coconstruction entre un mouvement cherchant l'attention des médias grand public et des journalistes en quête de la «bonne image».

Lors de la première phase de médiatisation, une contradiction s'établit par conséquent entre la sexualisation et la dépolitisation du corps des Femen dans la presse et le combat féministe qu'elles disent mener hors du champ médiatique. On se trouve face à un paradoxe : ce féminisme soudainement prisé par les médias, l'est en contraste avec un pays altérisé et n'échappe pas lui-même à des formes d'ethnoracialisation, par le biais d'une exotisation des membres du mouvement. C'est d'ailleurs bien là une des conditions de son accès à la sphère publique : le féminisme de Femen est valorisé dans les discours de presse par 
son combat contre une Ukraine présentée comme résolument sexiste, et ses militantes légitimes à prendre la parole en tant qu'elles répondent aux critères d'une féminité idéalisée. Cette première phase de médiatisation se caractérise donc par la répétition de normes hégémoniques de genre, par l'opposition constante entre modernité et archaïsme, Europe de l'Ouest et Europe de l'Est, émancipation et prostitution. Elle pose également les jalons de la construction médiatique de la figure mythologique genrée et ethnoracialisée qu'incarneront les activistes lors de leurs actions sur le territoire français : l' "amazone ».

\section{Les " amazones » face aux catholiques français}

L'arrivée à Paris de la leader Inna Shevchenko en août 2012 signe l'installation du collectif en France et marque le début de son événementialisation par les médias. Entre août 2012 et décembre 2013, la presse quotidienne et magazine consacre 250 articles à Femen. Cette médiatisation est marquée par des pics correspondant à ses différentes actions - de l'installation officielle au Lavoir moderne, dans le $18^{\mathrm{e}}$ arrondissement parisien, à la confrontation avec Civitas en passant par l' "affaire Amina » - et ne s'essouffle qu'après septembre 2013, avec seulement neuf articles d'octobre à décembre (graphique 2). L'arrivée du mouvement en France s'accompagne d'une structuration de sa communication sur le territoire national. Outre l'ouverture d'une page Facebook nourrie de façon quasi quotidienne et d'un compte Twitter permettant de suivre en direct les actions du collectif, la composition même du groupe, avec l'arrivée d'anciennes militantes du mouvement fortement médiatisé à la première moitié des années 2000, Ni putes ni soumises, telles Loubna Méliane et Safia Lebdi, et l'implication de militantes exerçant la profession de journaliste favorisent la mobilisation de réseaux de journalistes et la mise en œuvre de véritables campagnes de publicité (Bouton, 2015). On ne saurait pour autant réduire la médiatisation du mouvement à la seule volonté des intéressées, d'une part, parce que tous les mouvements féministes se dotant d'une stratégie de communication n'ont pas le même succès, d'autre part, parce que l'engouement médiatique semble avoir eu un effet d'entraînement dépassant Femen même. On peut, à titre d'exemple, évoquer le documentaire et l'ouvrage réalisés par la journaliste et essayiste Caroline Fourest sur le collectif, venue de son propre chef, mue par son intérêt revendiqué pour le féminisme. Un tel exemple montre combien le nombre important d'articles est aussi significatif d'une certaine valeur accordée par les commentateurs et journalistes au mouvement.

Le premier pic se situe autour de l'ouverture, le 19 septembre 2012, du quartier général et «premier centre d'entraînement» de Femen et pose les bases de ce qui constituera son image médiatique. C'est d'abord dans son usage des seins nus qu'il fait mouche. Ces derniers sont appréhendés comme 

Graphique 2 : Nombre d’articles parus par mois (tous journaux confondus)
lorsque Femen est territorialisé en France (août 2012 - décembre 2013)

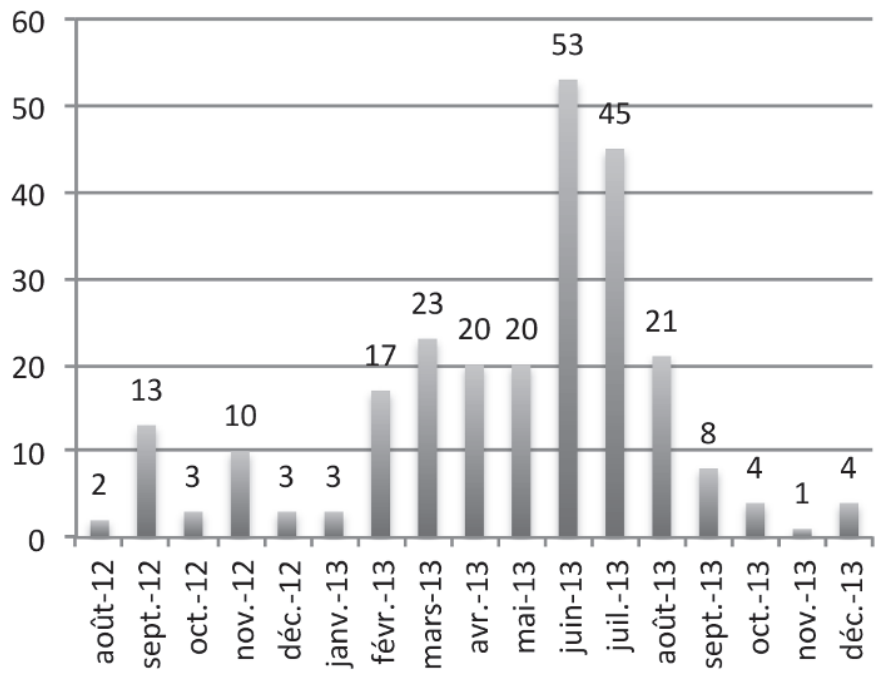

des symboles d'une prise de pouvoir. Ils se voient érigés, par la voix des militantes, en manifestation ultime de l'affranchissement de la domination masculine : pour le collectif, la nudité est symbole de réappropriation du corps féminin et de déstabilisation du patriarcat - «Pour la première fois, le corps des femmes n'appartient plus aux hommes. Ils sont décontenancés, ils ont peur », dira Inna Shevchenko dans Libération (18 septembre 2012). Mais les seins nus sont aussi perçus comme la marque d'une relation stratégique aux médias. "Arme de médiatisation massive» (Le Nouvel Observateur, 20 septembre 2012), «protection» recherchée (Libération, 18 septembre 2012), ils sont donnés à voir comme de véritables outils d'attraction des médias, ce qui contribue à rendre visible la mise en scène sous-tendant leur usage. Si une telle explicitation investit la couverture du mouvement d'une réflexivité journalistique, elle contribue à créer l'image d'un féminisme «moderne », «pop » - les termes sont récurrents dans l'ensemble de la presse - en tant qu'il ferait un emploi «décomplexé» du corps, c'est-à-dire d'apparence détaché des affects et de l'anticipation de ses significations (notamment sexuelles), et en tant qu'il serait porté par des militantes quasi professionnelles, maîtrisant les outils de communication. Elle vient ainsi parfaire l'image d'avant-garde du mouvement et nourrir une certaine conception de l'émancipation. Celle-ci devient synonyme d'usage «conscient» et « libre » de la nudité, autrement dit, d'un usage qui ne se préoccupe pas de la possible sexualisation du corps sous le regard 
masculin (Dalibert, Quemener, 2014), construisant une figure à rebours de la «féministe rabat-joie » évoquée par Sara Ahmed (2012).

C'est néanmoins l'ouverture du mariage aux couples de même sexe qui donne l'occasion d'un repositionnement du sens des actions de Femen dans le débat public français. Deux actions contribuent à une forte visibilité médiatique, la confrontation avec le groupuscule d'extrême droite Civitas lors d'une manifestation des «anti-mariage » le 18 novembre 2012, et l'action à NotreDame de Paris le 13 février 2013, au cours de laquelle des militantes célèbrent la démission du pape en criant «Pope no more» et sonnant les cloches de la cathédrale. Cette promotion au rang d’événement médiatique (Neveu, Quéré, 1996) s'appuie sur une mobilisation progressive de l'ensemble de la presse: si l'installation de Femen à Paris et l'altercation avec Civitas retiennent surtout l'attention de la presse de gauche et de centre-gauche (Libération, L'Humanité, Les Inrockuptibles, Le Nouvel Observateur), l'action à Notre-Dame, par la controverse qu'elle provoque (quarante articles sont publiés en février et mars 2013, contre six les deux mois précédents), mobilise la presse de gauche et de centre-gauche (quinze articles) comme la presse de droite et de centredroit, notamment Le Figaro et La Croix (vingt-cinq articles). Au cœur de cette phase de la médiatisation se tient un nouvel antagonisme, opposant Femen aux tenants et symboles de la religion catholique, ceux-ci se substituant à l'Ukraine dans les cibles du mouvement (tableau 1). Cet antagonisme s'inscrit dans une généalogie révélatrice de la dimension conjoncturelle de l'image des activistes. Si la cible religieuse est présente dans le discours de Femen dès sa création en Ukraine, elle est, dans les premiers temps de la médiatisation en France, peu valorisée au profit de portraits des membres et d'articles sur la «méthode» des seins nus. Mais elle se retrouve sur le devant de la scène avec le lancement du débat autour du «mariage pour tous» - cité dix fois lors de la période ukrainienne, le christianisme apparaît comme le « responsable » privilégié de la situation des femmes, avec soixante-quinze occurrences entre août 2012 et mars 2013 (tableau 1). Le discours antireligieux de Femen rencontre alors les agendas politique et médiatique en ce qu'il s'oppose au répertoire de la morale religieuse mobilisé par les opposants à la loi de la «Manif pour tous».

Tantôt disqualifiée, tantôt louée, cette opposition de Femen à la religion catholique structure les opérations de légitimation ou au contraire de discrédit du mouvement au sein d'une presse elle-même divisée autour de la question (Cervulle, 2014). La presse de gauche et de centre gauche assimile le catholicisme, par la voix des militantes, à ses branches les plus extrémistes, incarnées par des groupes tel Civitas, et à l'institution de l'Église (Le Monde, 7 mars 2013), tous deux étant érigés en menace sourde, « sexiste et rétrograde » (Ibid., 18 février 2013), pesant sur une France pourtant «émancipée» (Les Inrockup-

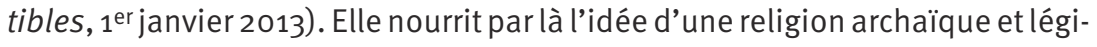
time en même temps les actions de Femen en France, qu'elle qualifie d'«anti- 
cléricales", inscrivant ainsi le mouvement du côté de la défense de la laïcité (Libération, 19 février 2013). À l'opposé de cette position conciliante, voire de cette célébration des actions de Femen, la presse de droite réprouve le collectif à la suite du happening de Notre-Dame de Paris, au nom de la défense de la mission civilisatrice de la religion chrétienne. La critique est particulièrement virulente au sein du Figaro, qui fait de Femen le symbole d'une «christianophobie» venant menacer la «civilisation» chrétienne (14 février 2013). Au sein du journal, l'antagonisme entre modernité et archaïsme se renverse à travers une réappropriation des termes ayant servi à disqualifier l'Église. Le pape Benoît XVI est ainsi présenté comme «l'inspirateur d'une contre-culture en marche, mobilisée contre les tyrannies et les pouvoirs abusifs» (15 février 2013). Ce renversement est le ressort d'un rejet total du mouvement, alors renvoyé au rang de "commando inqualifiable» et de «club de féministes plutôt mal famées» (14 février 2013).

À ces discours contradictoires répond un véritable storytelling (Salmon, 2007), dont la presse est la première à construire l'armature. Particulièrement sensible dans les articles de la presse magazine (Paris Match, Les Inrockuptibles, Le Nouvel Observateur) et de la presse quotidienne (Le Monde, Libération), cette mise en récit, structurée autour de quatre épisodes, s'appuie sur de nombreux discours rapportés de Femen servant à l'authentifier. La parole est ainsi très largement donnée aux membres du mouvement, comme le montre le numéro des Inrockuptibles du $1^{\mathrm{er}}$ janvier 2013, qui dédie deux pages entières au récit à la première personne de l'année 2012 par la leader Inna Shevchenko. La publication, en mars 2013, d'un ouvrage intitulé Femen, véritable manifeste revenant sur la création du mouvement (Ackerman, 2013), vient sceller cette narration médiatique. Elle brouille en outre les frontières entre le discours officiel de Femen et le discours journalistique, la presse relayant activement les moments forts de l'ouvrage. Le Monde publie ainsi dans son numéro du 7 mars 2013 les «bonnes pages» de l'ouvrage Femen, ouvrant au groupe un espace inédit de mise en récit des actions. Si un tel storytelling semble avoir tant fédéré, c'est aussi qu'il est une manière pour les médias de «faire sens» (Gitlin, 1980) avec un mouvement dont les membres se revendiquent davantage comme "praticiennes et non théoriciennes» (Le Nouvel Observateur, 4 avril 2013) et qui se constitue au moyen d'une performance permanente plutôt que par des discours. Durant les six premiers mois du collectif en France, il s'accompagne d'ailleurs d'une faible attention portée aux revendications sous-tendant des actions et s'élaborant à travers elles. Ces derniers se résument le plus souvent à un combat «contre le patriarcat» - l'expression, souvent explicite, reste très générale - et à la désignation d'une série de cibles, faisant écho aux slogans peints sur les torses, qui participent ici à la personnalisation excessive de la lutte. La seule référence théorique est la mention faite à l'ouvrage La Femme et le Socialisme d'August Bebel, désigné par la presse comme «l'ouvrage réfé- 
rence du groupe » (Libération, 18 septembre 2012) sans que soit explicitée la pensée qu'il développe.

La simplicité des messages favorise en outre un autre mode d'existence du mouvement, à savoir sa mise en images notamment par la presse magazine (Paris Match, Le Nouvel Observateur, Les Inrockuptibles) qui consacre des pages ou doubles pages aux photos de l'ensemble des militantes posant le regard dur, le torse nu et les poings serrés, ou de leurs corps en pleine action. La mise en récit prend alors tout son sens si l'on considère le revers d'une telle médiatisation : elle pallie en effet le possible manque d'intelligibilité des seules actions du mouvement en construisant un «cadre interprétatif» simple (Gamson, Modigliani, 1989), dans lequel Femen est rappelé à son origine ukrainienne, à l'engagement physique de ses membres et aux menaces subies pendant leurs actions. Elle contribue en outre à légitimer une lutte dirigée contre des cibles spécifiques (la prostitution, la dictature, la religion), en lui insufflant une trame narrative et une «identité».

La virulence des critiques, au même titre que le storytelling qui lui répond, renforce l'image d'un féminisme d'action, prêt à tout - et même à mettre en danger son intégrité physique - pour défendre une cause. Celle-ci s'élabore autour de la figure de l' " amazone », qualificatif utilisé par la presse? renvoyant à ces femmes guerrières, affichant une «fierté », une «force » et une « indépendance » par l'appropriation de méthodes viriles (Leduc, 2008). Présent dès les débuts du mouvement, cet imaginaire guerrier est certes le résultat d'une véritable «scénographie raisonnée », qui rend compte, selon Marie-Joseph Bertini, combien l'appareillage du mouvement dépend de sa médiatisation (Bertini, 2014). Mais il est aussi le produit d'un processus de signification par la presse qui illustre et désigne la part sacrificielle de l'engagement à force d'images de corps en action. Cet imaginaire guerrier sous-tend ainsi, dès juin 2012, le reportage photo de Paris Match portant sur une action contre la prostitution menée pendant l'Euro 2012 de football en Ukraine. On y voit les membres de Femen les poings en l'air, criant et brandissant des pancartes dans la foule, puis traînées à terre par un service d'ordre. Le reportage s'accompagne de qualificatifs courts qui insistent sur la lutte à la fois physique et politique : «militante », « résistante», «maîtrisée» (21 juin 2012). Il est suivi, le 19 septembre 2012, d’un reportage sur la «guérilla féministe» de Femen, présenté comme «à la pointe du combat» pour «mener la guerre du féminisme». Cette image de femmes guerrières, soutenues par des méthodes dites « révolutionnaires", « radicales » et «courageuses » se nourrit de la représentation d'un monde de violence et de danger, au sein duquel les activistes sont érigées en combattantes héroïques ${ }^{8}$. « Menacées de mort », «torturées » en Biélorussie (Les Inroc-

7. L'Express titre, le 10 août 2011, les «Amazones de Kiev».

8. La figure héroïque se construit à partir de l’idée de sacrifice, de martyre et de résistance dans le combat (Cassagnes-Brouquet, Dubesset, 2009). 
kuptibles, 10 septembre 2012), "prises à partie et rouées de coups» (Le Monde, 18 février 2013) ou encore "passées à tabac» par Civitas (Le Nouvel Observateur, 28 novembre 2012), elle concourt à créer des figures de martyres, justifiant la formation d'une "armée de femmes» et les méthodes «radicales, excessives, brutales» employées (Le Nouvel Observateur, 20 septembre 2012). Cet imaginaire guerrier construit activement l'image d'avant-garde, consacrée par

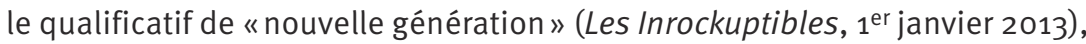
contrastant avec les «vieilles méthodes» (Les Inrockuptibles, 13 septembre 2012) d'un «féminisme de salon» (entretien avec Inna Shevchenko dans Le Parisien-Aujourd'hui en France, 24 mars 2013). Sa signification politique se déplace néanmoins lors de l'altercation avec Civitas et l'apparition de groupes, voire de contre-mouvements, tels que les Homen et les Antigones, chacun détournant à leur manière les modes d'action des activistes. Donnant l'image d'une véritable guerre de tranchées entre Femen et des "groupuscules» antimariage (Les Inrockuptibles, 18 avril 2013 et 30 mai 2013), l'ensemble de la presse finit par légitimer l'action du mouvement en élevant ce dernier au rang d'incarnation de la lutte pro-mariage et de participant à part entière du débat public. Dans ce contexte, les méthodes guerrières deviennent tout à la fois le corollaire de son efficacité et de sa dangerosité, cristallisant en grande partie les soutiens et critiques à l'égard du collectif.

\section{L’universel féministe face aux archaïsmes tunisiens}

Tableau 2. Liste des principaux syntagmes désignant et définissant les acteurs et/ou groupes pour qui Femen se mobilise dans sa médiatisation

\begin{tabular}{l|c|c|c|c}
\hline & $\begin{array}{c}\text { Période ukrai- } \\
\text { nienne octobre } \\
\mathbf{2 0 0 9 - j u i l l e t} \\
\mathbf{2 0 1 2}\end{array}$ & $\begin{array}{c}\text { Implantation } \\
\text { en France août } \\
\mathbf{2 0 1 2}-\text { mars } \\
\mathbf{2 0 1 3}\end{array}$ & $\begin{array}{c}\text { «Affaire } \\
\text { Amina } \\
\text { avril - août 2013 }\end{array}$ & $\begin{array}{c}\text { Baisse de la } \\
\text { médiatisation } \\
\text { septembre - } \\
\text { décembre 2013 }\end{array}$ \\
\hline $\begin{array}{l}\text { Femmes } \\
\text { «en général» }\end{array}$ & 0 & 21 & 27 & 4 \\
\hline $\begin{array}{l}\text { Femmes } \\
\text { ukrainiennes }\end{array}$ & 12 & 24 & 3 & 0 \\
\hline $\begin{array}{l}\text { Femmes } \\
\text { musulmanes }\end{array}$ & 0 & 4 & 59 & 0 \\
\hline Pussy Riot & 3 & 13 & 4 & 1 \\
\hline Amina Sboui & 0 & 3 & 596 & 1 \\
\hline $\begin{array}{l}\text { Personnes } \\
\text { LGBTQ }\end{array}$ & 0 & 1 & 0 & 0 \\
\hline
\end{tabular}


Il est néanmoins possible d'interroger les ressorts d'un tel déplacement dans la signification du mouvement. L'analyse des cibles du mouvement s'inscrit en effet en faux contre l'apparente territorialisation du combat Femen en France au travers d'une solidarité avec les mouvements LGBTQ. L'évaluation statistique des victimes à défendre citées lors de la médiatisation révèle d'abord une très faible attention à la cause gay, celle-ci n'étant mentionnée qu'à une seule reprise entre septembre 2012 et juin 2013 (tableau 2). De même, la situation spécifique des femmes en France n'est jamais directement mentionnée. Elle est partiellement effacée par une attention répétée portée à la cause des femmes ukrainiennes, citée vingt-sept fois, et des femmes en général, mentionnée à quarante-huit reprises entre août 2012 et août 2013. Comme nous le verrons, les femmes musulmanes deviennent quant à elles les «victimes » privilégiées dès avril 2013, avec cinquante-neuf mentions entre avril et août 2013. De tels résultats rendent non seulement compte de l'interchangeabilité des terrains de la lutte mais surtout d'une conception de plus en plus abstraite et détachée du contexte national de la victime du sexisme. L'image du mouvement se construit en outre au moyen d'une rhétorique hégémonique qui se manifeste par une énonciation distanciée : dans l'hexagone, les Femen ne parlent jamais en leur nom propre, elles parlent au nom des autres (les femmes ukrainiennes et, plus tard, les musulmanes) ou au nom d'un sujet du féminisme universel, homogène et impersonnel. Par cette rhétorique et la dénonciation d' "archaïsmes » situés dans des sphères éloignées de leur quotidien, cette image est le lieu non pas d'une confrontation au sexisme systémique en France, mais d'un évitement. Le féminisme tel qu'il est donné à voir dans la médiatisation du mouvement apparaît alors comme ne prenant pas position dans les rapports sociaux de genre à l'échelle locale, déniant par là la permanence de situations de domination et la nécessité même d'une lutte féministe en France. Il s'appuie par ailleurs, dans son discours, sur des processus d'altérisation qui contribuent à installer une "supériorité morale» (Grewal, 2005)9 et à conforter une position d' «exception» à l'échelle internationale de la France sur les questions de genre (Puar, 2013) ${ }^{10}$.

Ce déplacement vers un discours à la fois universaliste et altérisant se confirme avec la troisième période de notre corpus, consacré à l'" affaire Amina». Celle-ci, qui constitue le sujet principal des articles consacrés à Femen d'avril à août 2013, marque la territorialisation du discours journalistique en Tunisie et l'augmentation considérable de la publicité du mouvement : 159 articles sont publiés en cinq mois, dont de nombreux font la une des quotidiens et sont classées sous des rubriques à visée événementielle

9. Cette «supériorité morale» est une des modalités de production de la position hégémonique des féminismes internationaux, précise Grewal.

10. Cet «exceptionnalisme» est analysé par Jasbir Puar comme l'un des traits constitutifs de l'homonationalisme. 
(graphique 2). Elle démarre avec une photo postée sur Facebook d'une jeune femme poitrine nue, sur laquelle est peint le slogan « Mon corps m'appartient, il n'est l'honneur de personne ». Amina Sboui, à l'origine de la photo, fait dans son pays l'objet de plusieurs chefs d'accusation dont «atteinte aux bonnes mœurs» et "association de malfaiteurs». En France, l'affaire retient l'attention des quotidiens et hebdomadaires de gauche et de droite, qui voient en Amina la "première Femen tunisienne», et dans les conséquences familiales et juridiques de son geste la confirmation de l'archaïsme et du sexisme intrinsèque de la Tunisie. Une telle construction s'appuie dans un premier temps sur la description du comportement violent de la famille d'Amina Sboui en réaction à l'exposition publique de sa nudité : «la jeune femme, "pourtant majeure", serait mise "sous camisole chimique", comme le résume crûment Caroline Fourest. "Séquestrée et gavée de médicaments par sa famille [...]" " (Paris Match, 5 avril 2013). Elle s'appuie dans un second temps sur la réprobation générale du geste d'Amina au sein de la société tunisienne. Libération fait par exemple état du manque de soutiens tunisiens, y compris dans le «camp moderniste» (Libération, 31 mai 2013). Les féministes tunisiennes, a priori alliées "évidentes», sont quant à elles citées comme réprouvant son acte: «Féministe proclamée [...] l'historienne Raja Bahri [...] est sans états d'âme. “Amina? Oh, là, là! Je condamne! Si c'était ma fille, je condamnerais aussi !” » (Marianne, 6 avril 2013). Le conservatisme de la société tunisienne ainsi mis en scène apparaît d'autant plus condamnable que l'action d'Amina Sboui est présentée comme un acte d'émancipation - son assimilation au mouvement Femen venant nourrir cette interprétation. À travers ces procédés, la presse tend à produire l'image de Tunisiens défendant des valeurs archaïques, incompatibles avec celles de la République française.

La médiatisation de l'« affaire Amina » donne par conséquent lieu à un nouvel antagonisme, produisant l'idée d'une rupture civilisationnelle entre l'Occident et les pays arabo-musulmans. Celle-ci se manifeste dans l'émergence d'une nouvelle figure ennemie, l'intégrisme islamique, particulièrement présent dans le discours des Femen venues soutenir par des actions seins nus, en Tunisie, la jeune femme emprisonnée. Les syntagmes les plus nombreux utilisés pour désigner l'adversaire des Femen, d'avril à août 2013, renvoient à la religion musulmane et se constituent notamment des partisans d'un islam politique : «les salafistes», « les jihadistes d'Ansar al-Charia », «les barbus» (tableau 1). Cette frontière ethnoraciale, qui rejoue le mythe du « choc des civilisations » de Samuel P. Huntington, se matérialise par ailleurs dans la construction discursive d'une opposition entre deux stéréotypes féminins : la jeune femme blanche, libre parce que dévêtue, face à la musulmane, soumise parce que voilée. Dans la presse, l'émancipation des femmes non blanches serait ainsi corrélée à la monstration de leur poitrine. Ce discours se retrouve dans les différentes interprétations faites de la portée politique de la photo d'Amina 
Sboui, ainsi que celle d'Aliaa Magda Elmahdy, jeune étudiante égyptienne ayant elle aussi posté une photo d'elle nue sur Internet en octobre 2011. Dans Libération, la féministe iranienne Maryam Namazie présente le corps nu comme un symbole de l'émancipation dans les pays musulmans : «Amina et Aliaa représentent un nouveau mouvement de la libération de la femme, et, dans ce combat face au voile et à l'islam, la nudité représente un enjeu important» (Libération, 12 juin 2013). De tels articles nourrissent l'image d'une Tunisie en pleine régression au niveau des droits des femmes, ainsi que d'une société minée de l'intérieur par la montée des extrémismes. Ils rendent compte des angoisses contemporaines quant à la place de l'islam dans le monde arabomusulman.

La médiatisation de l' "affaire Amina » s'accompagne de la création d'une figure repoussoir ethnoracialisée, celle de la musulmane voilée, renvoyant par contraste les Femen à une figure modèle de la féminité appartenant à l'imaginaire de la blanchité, celle de la femme libérée du joug de la domination masculine. Cette mise en scène amorcée lors du débat autour du mariage pour tous se confirme : ce qui est laissé en creux du récit médiatique est bien la situation des femmes blanches d'Europe de l'Ouest, qui ne se voient jamais distribuer le rôle de victimes. Alors que les journalistes expliquent l'engagement des Femen ukrainiennes et des Tunisiennes par leur expérience du sexisme, les activistes de nationalité française sont quant à elles qualifiées par leur parcours militant et associatif antérieur. Contrairement aux autres, les Femen d'Europe de l'Ouest ne sont jamais invitées à témoigner des rapports de pouvoir qu'elles subissent et font surtout office de porte-parole des femmes non blanches, à l'image des trois activistes européennes emprisonnées en Tunisie à la suite d'une action organisée en soutien à Amina Sboui. À leur libération, la presse retient surtout le témoignage que font ces dernières lors d'une conférence de presse consacrée à leur détention, des mauvais traitements que supportent les prisonnières tunisiennes ${ }^{11}$. Parallèlement à la couverture médiatique, Femen profite de l' «affaire Amina » pour se positionner en tant que mouvement féministe international, revendiquant le droit de porter un discours universel sur la cause des femmes, comme l'énonce Inna Chevtchenko dans Libération : «Il n'y a pas de "white feminism", il y a des droits universels. Pourquoi les droits des femmes au Maghreb seraient différents de ceux des Américaines ou des Européennes?» (Libération, 6 juin 2013). L'ethnocentrisme de cet « universel féministe » n'est que très peu critiqué dans les médias, alors qu'il l'est régulièrement par les féministes non blanches depuis les années soixante-dix aux États-Unis (Dorlin, 2008). Celui-ci est en effet accusé, d'une part, de renvoyer les minorités à une position subalterne en ne prenant pas en compte leur capacité à porter un discours sur eux-mêmes et leurs besoins et, d'autre part, d'as-

11. Voir l'article paru dans Paris Match le 27 juin 2013. 
socier implicitement l'émancipation féminine à la blanchité12. Seule une tribune libre rédigée par l'universitaire Sara Salem en fait état dans Le Monde (13 juin 2013) et une seule mention est faite, dans Les Inrockuptibles, au contremouvement Muslimah Pride Day, constitué par des musulmanes pour dénoncer la manière dont elles sont décrites par les Femen (Les Inrockuptibles, 6 avril 2013). Le départ d'Amina Sboui à la fin du mois d'août, accusant le mouvement d'islamophobie, ne change rien : la sphère médiatique interroge peu l'inscription du mouvement dans les rapports de race et la médiatisation demeure toujours importante.

La reconnaissance médiatique de Femen s’interrompt néanmoins après l' "affaire Amina », suite à la présentation à la Mostra de Venise le 4 septembre d'un documentaire intitulé «L'Ukraine n'est pas un bordel». Ce film, en suggérant qu'un homme ukrainien serait à la tête du collectif, conduit à décrédibiliser les actions des activistes aux yeux des journalistes. Ces derniers réagissent soit par des discours dépréciatifs, comme dans Le Figaro le 13 septembre 2013 où les militantes sont définies comme étant «articulées depuis l'Ukraine par un homme, un gourou » qui «tirait les ficelles de l'organisation», soit par une absence de médiatisation - qui est totale dans la presse magazine. Seulement huit articles sont publiés au mois de septembre dans la presse quotidienne et neuf d'octobre à décembre, la majorité étant des brèves (graphique 2). Ces dernières, rendant généralement compte de leurs actions ciblant l’Église, sont plutôt négatives à l'égard du mouvement, à la fois dans la presse de gauche et de droite. Les propos de politiques (dont ceux de l'ancien maire PS de Paris, Bertrand Delanoë) condamnant le happening d'une des militantes ayant mimé un avortement dans l'église de la Madeleine à Paris, sont notamment rapportés à plusieurs reprises en décembre au sein des quotidiens. Tandis que la responsabilité de l'islam dans la production du sexisme pointé par Femen tout au long de son existence fait consensus dans la presse, celle du christianisme suscite quant à elle un traitement beaucoup plus nuancé. Cette différence de traitement est significative de la légitimité dont bénéficie la religion catholique dans l'espace public (et politique) français, alors que l'islam est, à l'inverse, particulièrement déprécié.

La médiatisation de Femen montre que l'image du mouvement se structure dans l'opposition à plusieurs causes définies à partir de l'agenda médiatique et par le mouvement lui-même. À travers la mise en avant des «droits universels » des femmes, de la solidarité avec la cause LGBT et de l'opposition à l'Église catholique puis à l'islam, l'image de Femen relève d'une mission de conscientisation qui répond parfaitement à l’idéal républicain d’égalité, de laïcité et d'universalité. Ce qu'une telle médiatisation laisse néanmoins en creux,

12. Le débat sur le port du voile à l'école, qui a occupé l'espace médiatique français en 2003 et 2004, a cristallisé de telles critiques (Benelli et al., 2006). 
c'est l'ethnocentrisme et la blanchité du mouvement, particulièrement perceptible dans l'extension de la lutte aux pays du Maghreb. En produisant l'image d'une société divisée entre modernité/émancipation et archaïsme/patriarcat, la médiatisation du mouvement construit une forme légitime d'intervention féministe dans la sphère publique. Un féminisme qui, en faisant de la nudité un symbole d'émancipation, contribue à détourner le débat public d'une interrogation sur la permanence d'un sexisme et d'un racisme systémiques. Un féminisme qui par ailleurs, en ciblant des ennemis «clairement sexistes » - profiteurs de la prostitution, catholiques intégristes ou encore islamistes -, conforte la manière dont la société française se pense, à savoir au-dessus des rapports de domination en termes de genre et de race.

\section{Références}

ACKerman Galia, 2013, Femen, Paris, Calmann-Lévy.

Ahmed Sara, 2012, "Les rabat-joie féministes (et autres sujets obstinés)», trad. O. Bonis, Cahiers du Genre, ${ }^{\circ}{ }^{5}$, p. $77-98$.

Benell Natalie, Hertz Ellen, Delphy Christine, Hamel Christelle, Roux Patricia, FalQUET Jules éd., 2006, "Sexisme et racisme. Le cas français», Nouvelles questions féministes, $\mathrm{n}^{0} 25$.

BERTING Jan, 2001, «Identités collectives et images de l'autre. Les pièges de la pensée collectiviste », Hermès, $\mathrm{n}^{\circ} 30$, p. 41-58.

BERTINI Marie-Joseph, 2014, «Fragments d'une épistémologie de la domination. La geste de Femen, un dispositif socio-technique de communication à haute tension », Médias, transgressions et négociations de genre, Cahiers de la transidentité, Horssérie nº 1 , A. Alessandrin, L. Biscarrat, K. Espineira, M.-Y. Thomas éd., Paris, L'Harmattan, p.19-38.

Bonnafous Simone, 1991, L'immigration prise aux mots, Paris, Kimé.

Bouton Éloïse, 2015, Confession d'une ex-Femen, Paris, Éditions du moment.

BREkHus Wayne, 2005, "Une sociologie de l'“invisibilité”. Réorienter notre regard», Réseaux, no129-130, p. 243-272.

CASSAgnes-Brouquet Sophie, Dubesset Mathilde, 2009, "La fabrique des héroïnes », Clio. Histoire, femmes et sociétés, nº30, en ligne : 〈http://clio.revues.org/9353〉 (consulté le 16 mars 2016)

CEFAï Daniel, 2007, Pourquoi se mobilise-t-on? Les théories de l'action collective, Paris, La Découverte / MAUSS. Cervulle Maxime, 2013, Dans le blanc des yeux. Diversité, racisme et médias, Paris, Éditions Amsterdam.

- 2014, "Les controverses autour du "mariage pour tous" dans la presse nationale quotidienne. Du différentialisme ethno-sexuel comme registre d'opposition», L'Homme et la société, no189-190, p. 207-222.

Champagne Patrick, 1990, Faire l'opinion, Paris, Minuit.

DALIBERT Marion, 2014, "Le marquage socio-discursif de la race par le genre. Les 
“Roms", les Tunisiens, les Ukrainiens et les habitants des banlieues françaises dans les médias", Revue française des sciences de l'information et de la communication, $\mathrm{n}^{\circ} 4$, en ligne : 〈http://rfsic.revues.org/743〉 (consulté le 16 mars 2016).

DAlibert Marion, QUemener Nelly, 2014, «Femen. L'émancipation par les seins nus?», Hermès, no69, p.169-173.

De Rudder Véronique, Poiret Christian, Vourc'h François, 2000, L'inégalité raciste. L'universalité républicaine à l'épreuve, Paris, PUF. DoRLIN Elsa éd., 2008, Black feminism. Anthologie du féminisme africain-américain, 1975-2000, Paris, L’Harmattan.

DYER Richard, 1997, White, Londres, New York, Routledge.

- 2004, Le star-système hollywoodien, suivi de Marilyn Monroe et la sexualité, Paris, L'Harmattan.

Fillieule Olivier, Tartakowsky Danielle, 2008, La manifestation, Paris, Presses de Sciences Po.

FRASER Nancy, 2001, «Repenser la sphère publique. Une contribution à la critique de la démocratie telle qu'elle existe réellement», trad. M. Valenta, Hermès, nº 31, p.125-156.

Gamson William A., Modiglianı Andre, 1989, "Media discourse and public opinion on nuclear power. A constructionist approach», American Journal of Sociology, vol.XCV, $n^{0} 1$, p. 1-37.

GILL Rosalind, 2003, «From sexual objectification to sexual subjectification : the resexualisation of women's bodies in the media », Feminist Media Studies, vol. III, no 1, p. 99-106.

GITLIN Ted, 1980, The Whole World is watching. Mass Media and the Making and Unmaking of the New Left, University of California Press, Berkeley.

GreWALInderpal, 2005, Transnational America. Feminisms, Diasporas, Neoliberalisms, Durham, Duke University Press.

Hall Stuart, 2007, Identités et cultures. Politiques des Cultural Studies, M. Cervulle éd., trad. C. Jacquet, Paris, Éditions Amsterdam, p. 129-168.

- 1997a, "The work of representation», Representation. Cultural Representations and Signifying Practices, S. Hall éd., Londres, Thousand Oaks, New Delhi, Sage Publications, p.13-74.

— 1997b, "The spectacle of the "Other" ", Cultural Representations and Signifying Practices, S. Hall éd., Birmingham, The Open University, p. 223-291.

Honneth Axel, 2000, La lutte pour la reconnaissance, Paris, Le Cerf.

LEDUC Guyonne éd., 2008, Réalités et représentations des Amazones, Paris, L'Harmattan.

LEFÉBURE Pierre, LAGNEAU Éric, 2001, «Les mobilisations protestataires comme interactions entre acteurs sociaux et journalistes ", Les effets d'information en politique, J. Gerstle éd., Paris, L'Harmattan, p.55-81.

Molotch Harvey, LeSTER Marylin, 1996, «Informer. Une conduite délibérée de l’usage stratégique des événements », trad. M.-C. Gamberini, Réseaux, n075, p. 23-41.

MoRTUREuX Marie-Françoise, 1993, «Paradigmes désignationnels», Semen, n8, p.117-136.

Neveu Érik, 1999, «Médias, mouvements sociaux, espaces publics», Réseaux, nº 98 , p. $17-85$. 
NeVeu Érik, QuÉRÉ Louis, 1996, «Présentation. Le temps de l'événement», Réseaux, $n^{0} 75 \cdot$ p. 5-21.

Paveau Marie-Anne, 2013, "Ces corps qui parlent. 2 : La petite vertu discursive des Femen », La pensée du discours [Carnet de recherche], 〈http://penseedudiscours. hypotheses.org/?p=11603〉 (consulté le 17 juillet 2015).

PUAR Jasbir K., 2013, «Homonationalisme et biopolitique», trad. M. Cervulle, Cahiers du Genre, n5 54 , p. 151-185.

SALMON Christian, 2007, Storytelling. La machine à fabriquer des histoires et à formater les esprits, Paris, La Découverte.

VoIrol Olivier, 2005, «Les luttes pour la visibilité. Esquisse d’une problématique», Réseaux, nº129-130, p. 89-121. 